\title{
TRANSFERENCIA DE FUNCIONES SINTÁCTICAS ARTIFICIALES ENTRE ESTÍMULOS RELACIONADOS POR EQUIVALENCIA: UN ESTUDIO DE POTENCIALES CEREBRALES RELACIONADOS CON EVENTOS*
}

\section{TRANSFER OF ARTIFICIAL SYNTAX FUNCTIONS AMONG EQUIVALENCE-RELATED STIMULI: AN EVENT-RELATED BRAIN POTENTIALS STUDY}

\author{
Angel Tabullo $0^{* *}$, Alberto Yorio $0^{* * *}$, Silvano ZanUtto ${ }^{* * * *}$ Y AlejandRo Wainselboim ${ }^{* * * *}$
}

\begin{abstract}
*Trabajo financiado por el Consejo Nacional de Investigaciones Científicas y Técnicas (CONICET) y por el Proyecto Teorías y modelos para la investigación en neurociencias: Registros conductuales y fisiológicos asociados a tareas de aprendizaje de categorías, relaciones de analogía y seguimiento de reglas gramaticales (UBACYT 20020100100589).

${ }^{* *}$ Doctor en Medicina y Licenciado en Psicología. Becario Postdoctoral del Consejo Nacional de Investigaciones

Científicas y Técnicas (CONICET). Sarratea 760, (5501) Godoy Cruz, Mendoza. Argentina.

E-Mail: angeltabullo@ibyme.conicet.gov.ar

${ }^{* * *}$ Doctor en Medicina. Profesor Titular de la Cátedra II de Neurofisiología y de Práctica de Investigación Métodos

Psicofisiológicos en la Facultad de Psicología de la Universidad de Buenos Aires (UBA).

${ }^{* * * *}$ Doctor en Biología e Ingeniero Electrónico. Miembro de la Carrera del Investigador Científico del Consejo Nacional de Investigaciones Científicas y Técnicas (CONICET). Profesor Titular de la Cátedra de Redes Neuronales y Director

del Instituto de Ingeniería Biomédica de la Facultad de Ingeniería de la Universidad de Buenos Aires (UBA).

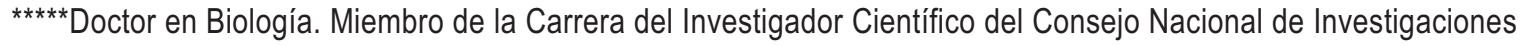
Científicas y Técnicas (CONICET).

Los autores agradecen a los directores del Laboratorio de Biología y Medicina Experimental, Doctor Enrique T. Segura

(a cuya memoria va dedicado este trabajo) y al Doctor Marcelo H. Cassini, el apoyo recibido y la colaboración permanentes para el desarrollo de la línea de investigación de este proyecto.
\end{abstract}

\section{RESUMEN}

La formación de clases de equivalencia entre estímulos ha sido propuesta en el campo del Análisis Experimental del Comportamiento como un prerrequisito conductual para el lenguaje. Adicionalmente, existe evidencia de que la transferencia de función entre estímulos equivalentes podría explicar la adquisición de estructuras sintácticas simples. No obstante, la simplicidad de las funciones sintácticas estudiadas no capturó la complejidad combinatoria de la gramática natural. Si la transferencia de funciones en clases de equivalencia es un modelo posible del desarrollo de estructuras gramaticales, debería ser verificado en contextos más válidos para el estudio del lenguaje. Los objetivos del trabajo realizado fueron los siguientes: (1) analizar la transferencia de funciones sintácticas en clases de equivalencia en un contexto válido para el estudio de la adquisición de reglas gramaticales, utilizando para ello el paradigma de gramáticas artificiales y (2) analizar los potenciales cerebrales relacionados con el procesamiento de esta transferencia de función, en secuencias gramaticales y no gramaticales. Se encontró evidencia comportamental de transferencia de función en un subgrupo de los sujetos experimentales. El potencial P600, típicamente asociado al costo de integración sintáctica en contextos lingüísticos, fue observado en estos sujetos ante: violaciones gramaticales con estímulos originales de la gramática artificial y secuencias con estímulos relacionados por equivalencia (gramaticales y no gramaticales). Se in- 
terpretó que el procesamiento de las secuencias artificiales implicó mecanismos neurobiológicos similares a los asociados a la sintaxis del lenguaje, y que el patrón de actividad P600 observado puede ser explicado por el aumento del costo de integración de los estímulos al contexto previo.

Palabras clave: Clases de equivalencia; Gramática artificial; Sintaxis; Transferencia de función; P600.

\section{ABSTRACT}

Stimulus equivalence class formation has been proposed as a behavioral prerequisite for language within the field of experimental analysis of behavior. Additionally, there is evidence that transfer of function among equivalent stimuli may explain acquisition of simple syntactic structures. However these experiments analyzed sequence func- tions that did not capture the complexity and versatility of natural grammar. If transfer of function between stimuli that belong to the same equivalence classes is indeed a useful model for the development of grammatical structures, then we should be able to verify it in a more valid context for the study of language. Artificial grammar learning tasks have been applied to the study of several aspects of language acquisition, from word segmentation to phrase structure and syntax rules. Furthermore, it has been shown that patterns of brain activity during processing of artificial grammars resemble those observed in language syntax processing. In particular, structural violations of language sentences and artificial grammar sequences both activate Broca's area. Therefore, artificial grammars provide a valid paradigm to study the learning of syntactic functions. The main objectives of the current work were: (1) to analyze transfer of function within equivalence classes in a valid context for the study of syntax acquisition, applying the artificial grammar paradigm and (2) to analyze brain potentials related to the transfer of function in grammatical and ungrammatical sequences. Fifteen subjects were trained to form two three-stimulus equivalence classes and then performed an artificial grammar learning task. One stimulus from each equivalence class was included as an item in the artificial grammar categories. During a test stage, subjects were asked to classify new artificial grammar sequences as grammatical or ungrammatical, while their EEG activity was registered. Half of these new sequences were built using the original training items and the other half contained equivalence-related stimulus. Subjects were assigned to two groups according to their performance in this test stage. Those participants whose percentage of correct responses was above $50 \%$ were considered to pass, while those below were assigned to the fail group. We found behavioral evidence of transfer of function in the pass subgroup. These participants were able to correctly discriminate grammatical from ungrammatical sequences that were built using original or equivalence-related stimulus. Event-Related potential Analysis of the EEG signal indicated a posteriorly distributed positivity with a topography and time-course similar to the $P 600$ potential. Within linguistic contexts, P600 is interpreted as the neural correlate of prediction and integration costs during syntax processing. It has been proposed that sentence comprehension depends on predictive mechanisms that combine lexical, semantic and syntactic information from linguistic input to anticipate future words. Processing of incoming stimuli is facilitated by pre- activation, allowing rapid integration to previous context. However, when the input does not match predictions, this integration becomes slower and more difficult, requiring additional neural resources. The P600 has been considered and index of increased integration costs, generated by unfulfilled predictions of word category and mor- phology based on previous context. In the present experiment, the P600 was observed after: grammar violations with the original artificial grammar lexicon and artificial sequences containing equivalence-related stimulus (both grammatical and ungrammatical). Results showed that artificial grammar processing involved neurobiological mechanisms that are similar to those associated in natural grammar processing. We interpreted the observed P600 pattern in terms of an increased stimulus integration cost, both in the case of grammatical and ungrammatical equivalence-related stimulus. Even though we consider that transference of function and equivalence class formations are by themselves insufficient to explain the complexity of natural grammar, we propose that this 
processes might be relevant to its acquisition and evolution, constituting a behavioral prerequisite for language development.

Key words: Equivalence classes; Artificial grammar; Syntax; Transfer of function; P600.

\section{INTRODUCCIÓN}

El paradigma de relaciones de equivalencia (Sidman \& Tailby, 1982) ha sido ampliamente utilizado en el campo del análisis experimental del comportamiento para el estudio de la formación de categorías y el comportamiento simbólico. Una relación de equivalencia se establece cuando, luego de entrenar una serie de asociaciones entre estímulos (típicamente por procedimientos de emparejamiento con la muestra o matching to sample - MTS), por ejemplo: A1-B1, B1-C1 y A2-B2, B2-C2, los sujetos son capaces de resolver relaciones nuevas, derivadas de las entrenadas, sin entrenamiento adicional. Estas relaciones cumplen las propiedades de la equivalencia tal como se define en Matemática y Lógica (reflexividad: A1-A1, B2-B2, etc., simetría: si A1-B1 $\rightarrow$ B1-A1, transitividad: si A1-B1 y B1-C1 $\rightarrow$ A1-C1 y simetría - transitividad combinadas: si A1-C1 $\rightarrow$ C1-A1). Esta última propiedad implica lógicamente a las anteriores, y generalmente se utiliza para evaluar más rápidamente la adquisición de una relación de equivalencia. Una vez que se verifican estas propiedades, se considera que los estímulos constituyen una clase de equivalencia (en el caso del ejemplo, se formarían dos clases de tres estímulos equivalentes cada una: A1-B1-C1 y A2-B2$\mathrm{C} 2$ ). Las relaciones de equivalencia resultan relevantes para el estudio de la formación de categorías debido a que no se basan en similitudes físicas o perceptuales entre los estímulos (que pueden incluso pertenecer a diferentes modalidades sensoriales) y no pueden ser explicadas por principios de discriminación y generalización tradicionales (Keller
\& Schoenfeld, 1950; Hall, 1996). Adicionalmente, los aprendizajes que involucran a un miembro de la clase (como un condicionamiento apetitivo o aversivo) se transfieren al resto de los estímulos equivalentes (Barnes, Smeets \& Leader, 1996; Roche \& Barnes, 1997). Este fenómeno se denomina transferencia de función y constituye una forma de generalización no restringida por las propiedades físicas.

Diversos aspectos de las clases de equivalencia han llevado a investigadores del campo del análisis experimental del comportamiento a plantear un posible vínculo con el desarrollo del lenguaje. Además del ya mencionado carácter arbitrario (es decir, independiente de propiedades físicas) de las relaciones aprendidas y emergentes, la naturaleza bidireccional de las asociaciones que se verifica en la propiedad simétrica se condisera un análogo de las relaciones significado / significante y símbolo / referente (Horne \& Lowe, 1996). Por otra parte, la formación de clases de equivalencia de estímulos no se ha observado en otras especies (Dickins, T. \& Dickins, D., 2001; Dugdale \& Lowe, 2000; Hayes, 1992) y se ha verificado consistentemente sólo en humanos lingüísticamente competentes, pero no en sujetos con trastornos en la adquisición del lenguaje o infantes preverbales (Augustson \& Dougher, 1992; Barnes, McCullagh \& Keenan, 1990; Devany, Hayes \& Nelson, 1986), lo que sugiere un vínculo entre ambas capacidades. Estos resultados han sido interpretados como evidencia de que la capacidad de formar clases de equivalencia constituye un prerrequisito conductual para el desarrollo del lenguaje (Sidman, 1986, 1994). Otros autores han extendido esta hipótesis, postulando que las relaciones de equivalencia serían el ejemplo más sencillo de una relación derivada entre estímulos (otros casos más complejos serían las relaciones derivadas de igualdad / oposición, o las relaciones de orden) y que las unidades comportamentales del lenguaje podrían definirse en términos de redes de relaciones derivadas entre estímulos. Esta propuesta se conoce como Teoría de los Marcos Relacionales (Hayes, BarnesHolmes \& Roche, 2001). 
Al aplicar las relaciones de equivalencia al estudio del lenguaje, se ha propuesto que las clases de equivalencia constituyen análogos comportamentales de la relación semántica de sinonimia (Chase, Ellenwood \& Madden, 2008; Imam \& Chase, 1988), dado que sus miembros son sustituibles en cuanto al control que ejercen sobre la conducta (de igual manera, al aprender que can significa lo mismo que perro, las representaciones y comportamientos asociados en la historia previa de aprendizaje a perro se transfieren automáticamente a su sinónimo). Por otro lado, se ha apelado a la transferencia de función en estímulos miembros de clases funcionales y clases de equivalencia para intentar explicar la adquisición de estructuras sintácticas simples, así como la posibilidad de construir una gran cantidad de frases nuevas a partir del entrenamiento con unas pocas palabras (Chase, Ellenwood \& Madden, 2008). Durante el aprendizaje de un idioma, el reforzamiento del uso de combinaciones de palabras como 'el perro ladra' o 'el auto corre' contribuye a la formación de clases funcionales entre los estímulos que ocupan la misma posición en cada frase (la clase de los artículos, la clase de los sustantivos y la de los verbos). Ahora bien, si se establece una relación de equivalencia entre un miembro de alguna de esas clases funcionales y una palabra nueva, el estímulo automáticamente pasa a utilizarse en la producción de secuencias nuevas, conservando la relación de orden con respecto a las otras clases adquiridas. Fenómenos de este tipo se han observado entre miembros de clases de equivalencia, luego de entrenar a los sujetos para formar secuencias simples con un subgrupo de los estímulos de cada clase. Luego de aprender a generar secuencias ordenadas de dos estímulos (B1,B2; B2,B3) con elementos de clases de equivalencia, los sujetos fueron capaces de producir secuencias nuevas transfiriendo las funciones de orden a estímulos no entrenados previamente (A1,A2; C2, C3) (Lazar, 1977). Otros estudios mostraron que es posible incluso entrenar cambios en el orden de las secuencias producidas a partir de claves contextuales (por ejemplo, agregar un tono como estímulo dis- criminativo para condicionar la producción de secuencias de tipo B1-B2-B3 o B3-B2-B1 (Lazar \& Kotlarchyk, 1986), incluso entre estímulos relacionados por transitividad a través de varios nodos (Wulfert \& Hayes, 1988; Chase et al., 2008). Estos resultados fueron interpretados como indicadores de que la transferencia de funciones sintácticas simples dentro de clases de equivalencia podía explicar la adquisición y generalización de combinaciones entre categorías gramaticales en el lenguaje.

Aunque prometedores, estos estudios se limitaron a entrenar la formación de secuencias extremadamente simples mediante procedimientos de condicionamiento operante. Por lo tanto, las funciones sintácticas estudiadas no capturaron la complejidad y versatilidad combinatoria de la gramática natural. Si la transferencia de funciones en clases de equivalencia es un modelo posible del desarrollo de funciones sintácticas, este fenómeno debería ser verificado en contextos más válidos para el estudio del lenguaje. Una posibilidad es usar para ello el paradigma de gramáticas artificiales (Reber, 1967). Una gramática artificial consiste en una serie de elementos (que pueden ser letras, fonemas, palabras o incluso estímulos visuales) y un conjunto específico de reglas para combinarlos en secuencias. En este tipo de tareas se presenta un número de secuencias construidas de acuerdo a estas reglas combinatorias, y luego del entrenamiento los sujetos son capaces de discriminar secuencias nuevas que cumplen con las reglas de la gramática de otras incorrectas, mostrando un rendimiento superior al azar en esta clasificación (Reber, 1989, 1993). Este resultado se observa aún cuando los sujetos no estén advertidos de la existencia de reglas en la estructura de las secuencias, y/o afirmen no haber aprendido ni aplicado ningún conocimiento al respecto. En ausencia de contenido semántico (como referentes asociados a las secuencias o significados previamente aprendidos sobre los estímulos) los sujetos sólo cuentan con información de tipo estadístico para aprender cómo los elementos pueden ordenarse y combinarse entre sí. Se han propuesto numerosas fuentes de infor- 
mación estadística que podrían explicar el desempeño en la tarea (Aslin, Saffran \& Newport, 1998; Fiser \& Aslin, 2002). De todas ellas, las más relevantes para la adquisición del lenguaje son las dependencias predictivas, patrones de co-ocurrencia que determinan la probabilidad de encontrar un elemento de una categoría a partir de la aparición previa de otro elemento (Saffran, 2001).

Las gramáticas artificiales se han utilizado para estudiar la adquisición de aspectos del lenguaje que van desde la segmentación de fonemas (Maye, Werker \& Gerken, 2002) y palabras (Peña, Bonnatti, Nespor \& Mehler, 2002; Saffran, Aslin \& Newport, 1996) hasta estructuras de frase y categorías sintácticas (Gómez \& Gerken, 1999; Saffran, 2002; Thompson \& Newport, 2007). Asimismo, estudios de neuroimagen funcional y electrofisiología señalan que los mecanismos neurales vinculados a la sintaxis del lenguaje también se asocian al procesamiento de gramáticas artificiales. Estudios de resonancia magnética nuclear funcional encontraron que la presentación de anomalías sintácticas en secuencias de gramáticas artificiales genera activación del área de Broca (Áreas de Brodmann 44/45) (Bahlmann, Schubotz \& Friederici, 2008; Petersson, Forkstam, \& Ingvar, 2004, Petersson, Folia \& Hagoort, 2012; Folia, Forkstam, Ingvar, Hagoort \& Petersson, 2011). Por otro lado, se observó que el potencial cerebral P600 (Osterhout \& Holcomb, 1992), asociado al costo de integración sintáctica (Kaan, Harris, Gibson \& Holcomb, 2000), fue generado por violaciones estructurales tanto en gramáticas naturales como artificiales (Christiansen, Conway \& Onnis, 2012; Tabullo, Sevilla, Segura, Zanutto \& Wainselboim, 2013), sin encontrarse diferencias entre ambos contextos.

Teniendo en cuenta la utilidad y validez del paradigma de gramáticas artificiales para el estudio de fenómenos sintácticos, el objetivo del trabajo realizado fue estudiar si las asociaciones predictivas adquiridas en el contexto de una gramática artificial se transfieren entre estímulos pertenecientes a una misma clase de equivalencia. Adicionalmente, la pre- gunta de investigación fue si los potenciales cerebrales asociados al procesamiento de estos estímulos equivalentes en secuencias gramaticales y no gramaticales serían similares a los evocados por el léxico original de la gramática. Se espera que, luego de haber verificado la formación de clases de equivalencia, y de haber incluido a un subgrupo de elementos en las categorías sintácticas de una gramática artificial, los sujetos sean capaces de sustituir esos elementos por estímulos equivalentes, discriminando adecuadamente entre secuencias gramaticales y no gramaticales construidas con estos nuevos estímulos. Asimismo, se espera que los potenciales cerebrales asociados al procesamiento de las secuencias sean similares entre estímulos originales y relacionados por equivalencia: los estímulos originales y equivalentes en posiciones incorrectas deberían generar un P600 con respecto a estímulos originales y equivalentes en secuencias gramaticalmente correctas.

\section{MÉtodo}

\section{PARTICIPANTES}

Participaron del experimento 15 sujetos adultos sanos diestros ( 8 mujeres, rango de edad: $20-28$, promedio de edad $24.3 \pm 4.2$ años), sin antecedentes de trastornos mentales o neurológicos. Todos los sujetos eran estudiantes de la Práctica de Investigación "Métodos Psicofisiológicos" de la Facultad de Psicología de la UBA y participaron voluntariamente del experimento. El protocolo experimental fue aprobado por el comité de ética del Instituto de Biología y Medicina Experimental (IBYME) y se siguieron estrictamente las normas internacionales para la investigación en humanos.

\section{PRocedimiento}

El experimento se llevó a cabo en una sesión, en la cual se presentaron las tareas a los sujetos en el siguiente orden: tarea de rela- 
ciones de equivalencia (entrenamiento y test), entrenamiento de gramática artificial, test de gramática artificial con estímulos originales y relacionados por equivalencia (realizado con registro EEG).

\section{1.- TAREA DE RELACIONES DE EQUIVALENCIA}

En la fase de entrenamiento, los participantes aprendieron cuatro relaciones basales entre estímulos mediante procedimientos de emparejamiento con la muestra (Matching to sample - MTS). Se les explicó que verían aparecer un estímulo de muestra en la parte superior de la pantalla de la computadora y dos de comparación en la parte inferior (ver Figura 1) y que tendrían que elegir cuál de éstos le correspondía al estímulo de muestra, presionando las teclas Ctrl izquierda y derecha. Se aclaró que los estímulos serían palabras artificiales carentes de significado, que la relación entre ellas era arbitraria, y que una vez realizada su elección el programa les informaría si había sido correcta. Se les pidió que respondieran los más rápido posible, tratando de no equivocarse. Se utilizaron las siguientes palabras artificiales como estímulos en el estudio: koza (A1), bare (B1), fita (C1), geki (A2), siro (B2), zofe (C2).

Se eligió un protocolo de entrenamiento de tipo Muestra como Nodo (también conocido como Uno a Muchos) debido a que se ha observado que aumenta las probabilidades de los sujetos de formar exitosamente las clases de equivalencia (Arntzen, Grondahl \& Eilifsen, 2010; Arntzen \& Holth, 1997, 2000). El procedimiento consiste en entrenar las asociaciones utilizando a los mismos estímulos como muestra en todos los ensayos. De esta manera, el entrenamiento se dividió en tres bloques: relaciones $\mathrm{AB}$ (A1-B1, A2$\mathrm{B} 2)$, relaciones $\mathrm{AC}(\mathrm{A} 1-\mathrm{C} 1, \mathrm{~A} 2-\mathrm{C} 2)$ y un bloque final en el que se intercalaron en forma semialeatoria ensayos de entrenamiento de las relaciones $\mathrm{AB}$ y $\mathrm{AC}$. Los dos primeros bloques tuvieron una duración de 16 ensayos cada uno y fueron presentados en orden contrabalanceado a los sujetos. El último bloque tuvo una duración de 64 ensayos. Cada blo- que fue precedido por una pantalla que sintetizaba las instrucciones del experimento. La estructura de los ensayos del entrenamiento se describe en la Figura 1.

Concluida la fase de entrenamiento, los sujetos continuaron con la fase de test de la tarea de equivalencia (Figura 1), en la cual debieron resolver un bloque de 32 ensayos de relaciones derivadas de simetría y transitividad combinadas: BC (B1-C1, B2-C2) y CB (C1-B1, C2-B2). Se les explicó que la presentación de los estímulos y las instrucciones eran las mismas que durante el entrenamiento, pero que ahora no se les informarían aciertos ni errores. Adicionalmente, se aclaró que las relaciones podían ser resueltas con los conocimientos adquiridos durante el entrenamiento. La verificación de la relación de simetría-transitividad combinada en los sujetos se consideró como evidencia de la adquisición de dos clases de equivalencia de tres estímulos cada una.

\section{2.- TAREA DE GRAMÁTICA ARTIFICIAL}

Se diseñó una gramática artificial con un léxico de 16 palabras artificiales, fonotácticamente correctas. Éstas podían ser monosílabicas ( $b a, d u, n e, g e, c e, l i, r i, k i)$ o bisilábicas, con una estructura consonante-vocalconsonante-vocal (bane, pefa, lane, fade, siro, rivo, soti, voni). Las secuencias de la gramática se construyeron a partir de dos estructuras posibles: (1) ba-categoría A (bare, pefa, lane, fade) - categoría $\mathrm{C}$ (ne, ge, ce) - categoría B (siro, rivo, soti, voni), (2) $d u$ - categoría B (siro, rivo, soti, voni) - categoría D (li, ri, ki) - categoría A (bane, pefa, lane, fade). Cada secuencia se construyó empezando siempre por $b a$ o $d u$ y continuando con un elemento de la categoría correspondiente. Un ejemplo de secuencia con estructura 1 sería: ba lane ge soti; mientras que una secuencia con estructura 2 sería: du rivo li pefa. Las categorías A y B de la gramática estuvieron construidas de forma tal que un elemento de cada una formaba parte de las relaciones de equivalencia de la tarea anterior. Así, el estímulo B1 - bare - pertenecía a la ca- 
tegoría A de la gramática artificial, mientras que el estímulo B2 - siro - pertenecía a la categoría B.

Antes de comenzar se les explicó a los sujetos que verían oraciones construidas con un lenguaje inventado, con palabras y reglas de carácter artificial y arbitrario. La tarea consistió en dos bloques sucesivos de entrenamiento por exposición pasiva, intercalados con dos bloques de entrenamiento con feedback correctivo. Estos últimos fueron incluidos debido a que un experimento previo (Arismendi et al., 2012) mostró que el rendimiento posterior de los sujetos mejora cuando son entrenados de esta manera. En los bloques de entrenamiento por exposición pasiva, los sujetos debieron prestar atención a las secuencias, respondiendo a intervalos aleatorios acerca de la presencia de un determinado ítem en la secuencia que acababan de ver (por ejemplo: "¿Apareció bare en la secuencia anterior?"). En cada uno de estos bloques se presentaron 40 secuencias construidas de acuerdo a las reglas de la gramática (20 por cada tipo de estructura), de izquierda a derecha en la pantalla de la computadora y de a una palabra por vez, con un intervalo de $200 \mathrm{~ms}$ entre la aparición de cada palabra. Una vez presentada la secuencia, permanecía en la pantalla durante dos segundos antes de pasar a la siguiente. En los bloques de entrenamiento por feedback se indicó a los sujetos que decidieran según su intuición si las secuencias presentadas obedecían a las reglas del lenguaje inventado o contenían violaciones, y recibieron feedback en cada ensayo de acuerdo a su respuesta. Las violaciones consistieron en todos los casos en presentar palabras en posiciones incorrectas. En cada bloque se presentaron 30 secuencias, 15 gramaticalmente correctas y 15 con violaciones. El formato de presentación de las secuencias fue el mismo que en las fases de entrenamiento pasivo, con la única diferencia de que esta vez las secuencias permanecían en la pantalla hasta que los sujetos realizaran su respuesta. La estructura de los ensayos de ambos bloques se describe en la Figura 1. Una vez concluido el entrenamiento en la ta- rea de gramática artificial, los sujetos continuaron con la fase de test del experimento con registro EEG.

\section{3.- TAREA del Test de TRansferencia de Fun- CIONES SINTÁCTICAS}

Se dieron instrucciones similares a las de las tareas anteriores, explicando que verían nuevas secuencias, algunas de ellas construidas de acuerdo a las reglas de la gramática y otras con violaciones de sintaxis y que deberían decidir si cada secuencia presentada era gramatical o no, presionando un botón al final de la secuencia. Adicionalmente, se explicó que podrían aparecer en algunas secuencias 'palabras' artificiales provenientes de la primera tarea y que su presencia podía ser o no incorrecta de acuerdo a lo que habían aprendido previamente. Por último, se aclaró que trataran de utilizar su intuición y que no pensaran demasiado sus respuestas.

Las secuencias fueron presentadas en esta tarea de a una palabra por vez, en el centro de la pantalla (en lugar de izquierda a derecha, para evitar artificios en el registro EEG debidos al movimiento ocular durante la lectura), con una duración de $500 \mathrm{~ms}$ por palabra y un intervalo entre palabras de $600 \mathrm{~ms}$. Por lo tanto, la asincronía de inicio de estímulos fue de $1100 \mathrm{~ms}$. Al final de cada secuencia, se presentó un signo '?', indicando que los sujetos debían responder en ese momento si la secuencia cumplía o no con las reglas de la gramática (ver Figura 2). El intervalo entre ensayos durante la tarea fue de 2 segundos.

En esta etapa se presentaron 160 secuencias, 80 gramaticales y 80 violaciones sintácticas. La mitad de las secuencias gramaticales fue construída con el léxico original de la gramática artificial y en la otra mitad se reemplazó en una de las categorías sintácticas una palabra artificial por otra relacionada por equivalencia. De igual forma, la mitad de las violaciones sintácticas se construyeron con los estímulos originales y en la otra mitad éstos fueron reemplazados por una palabra artificial que formaba parte de la misma clase 
de equivalencia (ver Tabla 1). Por lo tanto, se presentaron cuatro tipos de secuencias durante el testeo: gramatical con estímulos originales, gramatical con estímulos equivalentes, violación con estímulos originales y violación con estímulos equivalentes. Durante esta etapa se realizó el registro EEG, sincronizado con la aparición de las palabras erróneas en las violaciones sintácticas y sus correspondientes correctas en las secuencias gramaticales.

\section{4.- REGISTRO EEG Y ANÁLISIS ESTADÍSTICOS}

La actividad electroencefalográfica se registró con un equipo AKONIC BIOPC y un gorro Electro-Cap de 19 electrodos distribuidos de acuerdo al sistema internacional 10/20. La impedancia de los canales se mantuvo por debajo de $10 \mathrm{k} \Omega$. La señal de EEG fue digitalizada a $256 \mathrm{~Hz}$ y se aplicó un filtro digital entre los 0.5 y los $30 \mathrm{~Hz}$. Para cada tarea experimental se sincronizó el registro EEG con la aparición de los estímulos críticos. El análisis de los potenciales cerebrales relacionados con eventos se realizó mediante el software EEGLAB (Delorme \& Makeig, 2004). Se determinó una longitud de época de 2 segundos y una línea de base de $200 \mathrm{~ms}$ previos al inicio de la época. Los artefactos oculares se detectaron y removieron del trazado a través del algoritmo ADJUST (Mognon, Jovicich, Bruzzone \& Buiatti, 2010), basado en el análisis de componentes independientes de la señal (Independent Component Analysis - ICA). Las épocas que contenían otros tipos de artefactos fueron detectadas por inspección visual y eliminadas manualmente. Solamente aquellos ensayos en los que los sujetos respondieron correctamente fueron incluidos en el análisis.

Para el análisis estadístico de los potenciales cerebrales relacionados con eventos, se calculó el voltaje promedio dentro de las ventanas temporales de interés, determinadas a partir de la inspección visual y de resultados publicados previamente. El voltaje promedio se analizó mediante ANOVAS de medidas repetidas $3 \times 5 \times 2 \times 2$, que incluyeron los si- guientes factores intrasujeto: Región del electrodo (anterior, central, posterior), Posición lateral del electrodo [cinco regiones de interés, de izquierda a derecha: (1) electrodos F7, T3 y T5, (2) electrodos F3, C3 y P3, (3) electrodos Fz, Cz y Pz, (4) electrodos F4, C4 y P4 y (5) electrodos F8, T6 y P8)], Tipo de estímulo (original, equivalente) y Gramaticalidad de la secuencia (gramatical, violación). Se aplicó la corrección GreenhouseGeisser para las violaciones de esfericidad y el ajuste de Bonferroni para las comparaciones post hoc. El tamaño de efecto se estimó mediante el coeficiente eta cuadrado parcial. El análisis estadístico de los datos se llevó a cabo mediante el software SPSS v15.

\section{RESULTADOS \\ DATOS COMPORTAMENTALES}

Los sujetos fueron organizados de acuerdo a su desempeño en la fase de test de transferencia de funciones sintácticas en dos grupos: aquellos que superaron la tarea $(n=9$, porcentaje de aciertos superior al 50\%) y aquellos que no la superaron $(n=6$, porcentaje de aciertos por debajo del $50 \%$ ). El rendimiento del grupo que superó esta fase estuvo significativamente por encima del nivel de azar $(T(8)=3.471, p=.008)$. El desempeño de ambos grupos en las diferentes fases del experimento (1: Entrenamiento en equivalencia, 2: Entrenamiento en gramática artificial, 3: Test de transferencia de funciones sintácticas) se informa en la Tabla 2.

Se comparó mediante test de t para muestras independientes el porcentaje de aciertos de los sujetos en las diferentes fases del experimento. Si bien el grupo de sujetos que superó la tarea tuvo en promedio un mejor rendimiento en los entrenamientos de equivalencia y gramática artificial, esta diferencia no fue significativa en ninguno de los casos, y sólo alcanzó el nivel de significación en el test de transferencia de funciones sintácticas $(T(13)=2.901, p=.012)$.

A fin de analizar el desempeño de ambos grupos en el test con mayor profundidad (ver 
Tabla 3), se realizó un ANOVA mixto $2 \times 2 \times 2$ con Gramaticalidad (gramatical, violación sintáctica) y Tipo de estímulo (original, equivalente) como factores intrasujeto y Grupo como factor intersujeto. Se encontraron efectos principales significativos de Grupo $[F(1$, $\left.13)=8.192, p=.013, \eta^{2} \mathrm{p}=.387\right]$, Tipo $[F(1$, $\left.13)=14.413, p=.002, \eta^{2} \mathrm{p}=.526\right]$ y Gramaticalidad $[F(1,13)=8.332, p=.013$, $\left.\eta^{2} \mathrm{p}=.391\right]$, así como una interacción significativa entre ambos $(F=4.883, p=.046$, $\eta_{\mathrm{p}}^{2}=.273$ ). El desempeño de los sujetos fue mejor para los estímulos originales que para los relacionados por equivalencia en las secuencias gramaticales $(p<.001)$ y mejor para las secuencias gramaticales que para las violaciones con estímulos originales $(p=.011)$. Cabe destacar que el grupo de sujetos que superó la tarea tuvo en promedio un desempeño superior al azar tanto en las secuencias con estímulos originales como en las que tenían estímulos relacionados por equivalencia, lo cual indica que fueron capaces de transferir la función sintáctica entre los miembros de una clase de equivalencia.

POTENCIALES CEREBRALES RELACIONADOS CON EVENTOS

Sólo se consideraron para este análisis los datos del grupo de sujetos que superó la fase del test del experimento. La inspección visual de los promedios generales de los trazados EEG indicó la presencia de una positividad posterior entre los 500 y $700 \mathrm{~ms}$ luego de la palabra crítica en las violaciones sintácticas y en las secuencias gramaticales con estímulos equivalentes. Por su latencia y distribución topográfica este potencial es comparable con los efectos P600 observados en experimentos previos (Christiansen et al., 2012; Tabullo et al., 2013). El curso temporal de la positividad fue más prolongado para las secuencias gramaticales con estímulos equivalentes. Cabe destacar que esta positividad estuvo ausente en el grupo de sujetos que no superó la tarea. Se determinaron dos ventanas temporales de interés para el análisis estadístico del potencial: de 500 a $600 \mathrm{~ms}$ y de 600 a $700 \mathrm{~ms}$. La Figura 3 muestra el promedio general de los trazados por condición experimental en seis canales representativos. La Figura 4 muestra el promedio general ampliado en un canal representativo y las topografías correspondientes a las ventanas previamente descriptas. Los resultados de los análisis se describen a continuación.

\section{VENTANAS TEMPORALES ANTERIORES AL POTENCIAL}

Con el fin de verificar que no existieran diferencias significativas en el trazado previo a la positividad de interés, se realizó el análisis de la señal en tres ventanas tempranas: línea de base (-200 ms hasta el momento 0 de la época), 0 a 200 ms y 200 a 400 ms). No se encontraron efectos principales ni interacciones significativas en ninguno de estos períodos (línea de base: F's < 1.315, p's > .289; $0-200$ ms: F's < 1.523, p's > .219; 200 a 400 ms: F's $<1.164$, p's $>$.320).

\section{P600 - VENTANA TEMPRANA}

Se encontró una interacción Posición Lateral $\times$ Tipo $\times$ Gramaticalidad $[F(4$, $\left.32)=3.369, p=.021, \eta_{p}^{2}=.296\right]$. En las secuencias con estímulos originales, las violaciones fueron más positivas que las secuencias gramaticales en los canales izquierdos $(p=.029)$ y los estímulos gramaticales relacionados por equivalencia fueron más positivos que los originales, también en los canales izquierdos $(p=.041)$.

\section{P600 - VENTANA TARDÍA}

Se encontró una interacción Región $\times$ Tipo $\times$ Gramaticalidad $[F(2,16)=7.017$, $\left.p=.006, \eta_{\mathrm{p}}^{2}=.467\right]$. En las secuencias gramaticales, los estímulos equivalentes generaron positividades mayores que los ori- 
ginales en los canales posteriores $(p=.001)$. Adicionalmente, las secuencias gramaticales con estímulos equivalentes fueron más positivas que las no gramaticales con estímulos equivalentes $(p=.001)$ en los canales posteriores.

\section{Discusión}

Los resultados comportamentales del experimento aportaron evidencia de transferencia de funciones sintácticas artificiales entre los miembros de una clase de equivalencia en un subgrupo de los participantes. Este tipo de transferencia de función se había observado hasta el momento con funciones sintácticas de orden simples (Chase et al., 2008; Cullinan, Barnes, Hampson \& Lyddy, 1994; Imam \& Chase, 1988; Lazar, 1977; Lazar \& Kotlarchyk, 1986; Wulfert \& Hayes, 1988), pero nunca en el contexto de una gramática artificial. Estudios anteriores mostraron que, luego de entrenar asociaciones por emparejamiento con la muestra en un grupo de estímulos (por ejemplo: A1-B1-C1 y A2-B2-C2) $\mathrm{y}$ aprender a producir secuencias con un subconjunto de ellos (por ejemplo: A1, A2; B1, $\mathrm{B} 2$ ), los sujetos fueron capaces de generar secuencias nuevas sin entrenamiento adicional, transfiriendo las funciones de orden al resto de los miembros de la clase (por ejemplo: $\mathrm{C} 1$, C2) (Lazar, 1977). Posteriormente, se observó que podían transferirse funciones de orden condicionales, controladas por claves contextuales entre estímulos miembros de clases de equivalencia (por ejemplo, utilizando diferentes tonos como estímulo discriminativo para determinar la direccionalidad de la secuencia: "B1, B2" o "B2, B1") (Lazar \& Kotlarchyk, 1986), incluso entre estímulos relacionados por transitividad a lo largo de varios nodos (Wulfert \& Hayes, 1988). Adicionalmente, se observó que secuencias de tres estímulos podían ser aprendidas y transferidas mediante estos procedimientos (Chase et al., 2008). Estos resultados fueron interpretados como evidencia de que la transferencia de función en clases de equivalencia de estímulos podía constituir un mo- delo para explicar el desarrollo de relaciones semánticas (sinonimia), clases sintácticas y transferencia de características sintácticas a estímulos nuevos (Chase et al., 2008). Sin embargo, estos experimentos se limitaron a estudiar funciones sintácticas simples (relaciones de orden en secuencias de dos o tres estímulos) que fueron entrenadas explícitamente. Hasta el momento no se había intentado estudiar la transferencia de propiedades sintácticas más complejas, en un contexto que reflejara mejor el aspecto implícito de la adquisición de la sintaxis del lenguaje. Por lo tanto, los resultados obtenidos extienden el alcance de los hallazgos previos, mostrando que los estímulos miembros de una clase de equivalencia transfieren entre sí las asociaciones predictivas entre categorías gramaticales adquiridas en el contexto de gramáticas artificiales.

Cabe destacar que si bien todos los sujetos entrenados verificaron la formación de clases de equivalencia, algunos de ellos fueron incapaces de transferir las expectativas estructurales de la gramática de los estímulos del léxico original a los equivalentes. Esto podría deberse a un aprendizaje insuficiente de dichas expectativas, dado que el rendimiento de estos sujetos durante el entrenamiento en gramática artificial fue inferior al de aquellos que sí superaron el test. Futuros estudios podrían determinar qué otros factores se asocian a la variabilidad interindividual en la transferencia de funciones sintácticas en clases de equivalencia.

En concordancia con los resultados comportamentales, el análisis de los potenciales cerebrales en los sujetos que superaron el test indicó un efecto P600 al comparar las secuencias no gramaticales (tanto con estímulos originales como con los relacionados por equivalencia) y las gramaticales con estímulos equivalentes, con las gramaticales originales. En contextos lingüísticos, el P600 es interpretado como el correlato neural del costo de procesos de predicción e integración durante el procesamiento sintáctico (Hagoort, 2009; Kaan, 2007, 2009; Kaan, Harris, Gibson $\&$ Holcomb, 2000). Se ha propuesto que la comprensión de oraciones depende de meca- 
nismos predictivos en los que la información lexical, semántica y sintáctica de las palabras percibidas se combina para anticipar las características de las probables palabras siguientes (Gibson, 1998; Hagoort, 2009; cf., De Long, Urbach \& Kutas, 2005; van Berkum, Brown, Zwitserlood, Kooijman \& Hagoort, 2005). Al preactivarse las características de las continuaciones más probables de la oración, se facilita el procesamiento de las palabras futuras, permitiendo que su integración se lleve a cabo en la escala de tiempo requerida por la velocidad del lenguaje oral. No obstante, cuando el input no se corresponde con las expectativas, esta integración se hace más lenta y difícil, requiriendo el reclutamiento de recursos neurales adicionales. El P600 sería el reflejo del incremento en el costo de integración sintáctica generado por el incumplimiento de predicciones sobre la categoría gramatical o morfología de las palabras, basadas en el contexto previo (Kaan et al., 2000). Por otro lado, se ha propuesto que los mecanismos predictivos asociados al P600 no están limitados al lenguaje, sino que constituyen procesos de dominio general que son reclutados para el procesamiento de la sintaxis (Christiansen et al., 2012; Kaan, 2007, 2009; Hagoort, 2009; Patel, Gibson, Ratner, Besson \& Holcomb, 1998). En el caso de las gramáticas artificiales, el P600 generado por violaciones sintácticas también se ha interpretado como producto del incumplimiento de expectativas basadas en asociaciones predictivas entre los elementos de las secuencias (Bahlmann, Gunter \& Friederici, 2006; Christiansen et al., 2012, Tabullo et al., 2013). Adicionalmente, se observó que estas asociaciones pueden ser de naturaleza probabilística, dado que combinaciones de estímulos más improbables, pero gramaticalmente correctas, generan P600 mayores que combinaciones de estímulos altamente esperadas (Tabullo et al., 2011).

A diferencia de lo planteado en las predicciones experimentales para este estudio, la positividad asociada a los estímulos equivalentes en secuencias gramaticales no fue similar al potencial evocado por los estímulos originales, sino que tuvo una mayor amplitud y duración en el tiempo que el P600 originado por las violaciones estructurales. A pesar de resultar contraintuitivo, este resultado podría explicarse apelando a la interpretación del P600 como un indicador del costo de integración del estímulo a la estructura previa. En las secuencias no gramaticales con estímulos originales, la aparición de un elemento en una posición incorrecta constituye una violación de la expectativa con respecto a la clase sintáctica esperada. Dado que los estímulos relacionados por equivalencia no han sido percibidos previamente por los sujetos como parte de las secuencias, al encontrar un estímulo equivalente en una posición gramaticalmente correcta, los sujetos deben ser capaces de evocar el estímulo original correspondiente y reconocer su pertenencia a la clase sintáctica esperada. Cualquier potencial predicción del sujeto sobre los estímulos equivalentes debe estar necesariamente mediada por los estímulos originales y sostenida por la relación de equivalencia. Esta diferencia generaría un costo de procesamiento adicional para integrar los estímulos al contexto previo de la secuencia, que se vería reflejado en el potencial P600. Un mecanismo similar fue propuesto para explicar el aumento en el potencial N400 que se observa al comparar pares de estímulos relacionados por equivalencia con pares directamente relacionados por el entrenamiento en un paradigma de priming (Barnes-Holmes et al., 2005). En este caso, la activación del estímulo prime se propaga con mayor intensidad a los miembros de la clase directamente relacionados, y en menor grado a estímulos relacionados a través de nodos intervinientes, generando para éstos menor facilitación y mayores costos de procesamiento.

Dado que los estímulos relacionados por equivalencia no pertenecen directamente a las clases sintácticas, ni forman parte del léxico original de la gramática, es posible que su aparición constituya otra violación a las predicciones que los sujetos realizan durante el procesamiento de las secuencias, generando así un P600 respecto de los estímulos gramaticales originales. Al encontrar un estímulo equivalente en una posición gramati- 
calmente correcta, los sujetos deben ser capaces de evocar el estímulo original correspondiente y reconocer su pertenencia a la clase sintáctica esperada. Otra posibilidad es que las predicciones sobre los posibles elementos futuros se propaguen también a los estímulos relacionados por equivalencia, pero con menor intensidad debido a la distancia nodal entre ellos de manera análoga a lo que ocurre con el potencial N400 durante el priming indirecto (Barnes-Holmes et al., 2005), con lo cual su reconocimiento y procesamiento resultan más difíciles. En cualquier caso, el costo de las operaciones adicionales necesarias para integrar los estímulos equivalentes al contexto previo de la secuencia se vería reflejado en el P600.

Esta interpretación podría explicar también la duración más prolongada de la positividad para los estímulos gramaticales equivalentes con respecto a los no gramaticales originales. Por un lado, puede pensarse que la violación de expectativa resulta mayor para los estímulos equivalentes, aún cuando estos están en la posición correcta, porque los no gramaticales originales sí forman parte del léxico de la gramática, y han sido observados ocupando posiciones similares en las secuencias durante el entrenamiento. En particular, las secuencias no gramaticales presentadas durante las fases de feedback pueden haber contribuido a generar en los sujetos expectativas sobre la posibilidad de encontrar violaciones similares durante el test (mientras que ninguna de las secuencias presentadas anteriormente incluyó estímulos equivalentes). Estudios previos han mostrado que la manipulación de la expectativa de los sujetos de encontrar una violación sintáctica puede reducir o suprimir el potencial P600 (Coulson, King \& Kutas, 1998; Hahne \& Friederici, 1999). Por último, los sujetos pueden reconocer más rápidamente que un estímulo original no pertenece a la clase sintáctica esperada, mientras que tienen que procesar la relación de equivalencia antes de poder integrar los estímulos equivalentes. Aunque no se tienen elementos para precisar la naturaleza de las operaciones involucradas, se puede afirmar que el costo cognitivo adicio- nal se vería reflejado en la mayor duración del potencial.

Podría argumentarse que los estímulos relacionados por equivalencia en posiciones gramaticalmente incorrectas debería haber generado el efecto P600 de mayor magnitud, dado que combinan la violación de expectativa sobre el léxico de la gramática y de la clase sintáctica correcta (o bien, porque reciben una facilitación mínima o nula por no pertenecer a la clase de equivalencia del estímulo esperable para dicha posición). No obstante, se observó un P600 similar para estímulos no gramaticales originales y equivalentes, mientras que el efecto se prolongó solamente para los gramaticales equivalentes. Este resultado podría explicarse asumiendo que, ante la dificultad extrema de integrar el estímulo a la secuencia, éste se procesa rápidamente como incorrecto; mientras que la posibilidad de ubicar los estímulos equivalentes en esa posición hace que el procesamiento y la actividad neural continúen por más tiempo. Se han observado resultados similares en un estudio que comparó violaciones de estructura de frase y de inflexión verbal en oraciones del español (Hinojosa, Martin-Loeches, Casado, Muñoz \& Rubia, 2003). Ambas violaciones generaron un P600 similar en una ventana temprana (500-600 ms), pero el efecto de la inflexión verbal se prolongó en una ventana posterior (600-700 ms). Los autores asociaron la duración adicional del potencial en esta condición a procesos de reparación sintáctica, que eran más factibles en el caso de las violaciones de inflexión verbal que para las violaciones de categoría (dado que éstas últimas producen una perturbación mayor de la estructura de la frase). Si bien los resultados obtenidos no pueden interpretarse en términos de procesos de reparación o reanálisis, sí coinciden en mostrar como el P600 puede prolongarse al comparar una estructura dudosa, pero potencialmente correcta, con otra claramente errónea, reflejando el reclutamiento de recursos neurales adicionales en el primer caso.

Por otra parte, es posible hallar un paralelismo entre los efectos P600 observados en nuestro experimento y los resultados de un estudio que analizó los potenciales cerebrales 
asociados a cambios de idioma en sujetos bilingües (Moreno-Montes, 2005; Moreno, Federmeier \& Kutas, 2002). En este trabajo se analizaron los PREs en sujetos bilingües (inglés - español) al comparar oraciones con tres finales posibles: (1) finales esperados, que consistieron en la palabra con mayor probabilidad de cierre (The ship dissapeared into the fog), (2) cambios léxicos, en los que se usaron sinónimos de las palabras esperadas (The ship dissapeared into the mist) y (3) cambios de idioma, en los que se presentó la traducción literal de la palabra esperada a la segunda lengua de los sujetos (The ship dissapeared into the niebla). Mientras que los cambios léxicos generaron un potencial N400, se observó un componente positivo tardío (late positive component - LPC) con distribución posterior para los cambios de idioma. Los autores interpretaron el LPC como indicio de que los cambios de idioma

“(...) puedan ser más bien tratados como eventos inesperados a nivel físico (ej., Kutas \& Hillyard, 1980; Osterhout, Bersick \& McLaughlin, 1997), y en consecuencia requieran mayores recursos para la evaluación del estímulo y la actualización en memoria (Kutas, McCarthy \& Donchin, 1977; Brookhuis, Mulder G., Mulder L., Gloerich, Van Dellen, van der Meere \& Ellermann, 1981; Donchin \& Coles, 1988; Sommer, Leuthold \& Matt, 1998). Esto apoya la hipótesis de que los costes asociados con el procesamiento de los cambios de idioma pueden surgir predominantemente en los estadíos de procesamiento relacionados con la toma de decisiones (ej., Thomas \& Allport, 2000)" (MorenoMontes, 2005, p. 55).

Si bien el potencial no fue considerado directamente por los autores como una instancia del P600, también afirmaron que

“(...) cierto tipo de eventos lingüísticos inesperados (e.j., cierto tipo de violaciones sintácticas) generan también positividades tardías similares (e.j., Coulson et al.., 1998; Münte, Matzke \& Johannes, 1997)" (Moreno-Montes, 2005, p. 53).

En el caso de nuestro experimento, la aparición de estímulos relacionados por equivalencia en las secuencias también constituyó un evento inesperado a nivel físico, dado que éstos no compartieron un contexto de aprendizaje con los elementos del léxico de la gramática. Si bien el léxico de la gramática artificial no es directamente comparable al de un lenguaje, el cambio de un idioma al otro podría ser una situación similar (en términos de violación de expectativa) al cambio de contexto entre estímulos aprendidos en la gramática artificial y en la tarea de relaciones de equivalencia. Una posible objeción a este razonamiento es que, si las relaciones de equivalencia son un análogo funcional de la relación semántica de sinonimia (Chase et al., 2008), entonces los estímulos equivalentes deberían producir un potencial cerebral similar al observado en los sinónimos en el experimento de Moreno-Montes (2005), y no al de las palabras de otro idioma. Sin embargo, los sinónimos están mucho más relacionados con el resto del léxico y son mucho más predecibles en el contexto de una oración del mismo idioma, que la palabra equivalente en otra lengua. En este sentido, los estímulos equivalentes dentro de las secuencias podrían estar generando un efecto de sorpresa similar al provocado por palabras de una lengua en el contexto de oraciones de otro idioma.

Cabe señalar como limitación del experimento realizado el reducido número de sujetos que pudo ser incluido en el análisis de los potenciales cerebrales. Si bien se observaron efectos significativos congruentes con las hipótesis experimentales, la relación señalruido fue baja en comparación a otros estudios con mayor cantidad de participantes. En consecuencia, se requiere interpretar con cautela los efectos observados a nivel del P600 y verificarlos en experimentos futuros con mayores tamaños de muestra.

En conclusión, el experimento mostró que los estímulos miembros de una clase de equivalencia pueden transferir asociaciones predictivas de categorías sintácticas de una 
gramática artificial a otros estímulos equivalentes. Adicionalmente, el potencial P600 asociado a esta transferencia de función es similar al registrado en otros estudios de lenguaje y gramáticas artificiales y puede ser interpretado en términos de costo de integración de los estímulos a la estructura previa. En este sentido, los resultados obtenidos son congruentes con estudios anteriores que mostraron transferencia de funciones sintácticas simples en clases de equivalencia. Estos hallazgos fueron interpretados anteriormente como evidencia de que

“(...) la simple sintaxis de frases de dos palabras puede dar lugar a frases de tres palabras y, a través de la formación de clases de equivalencia, a nuevas frases de dos y tres palabras. De esta forma, la transferencia de función a través de clases de equivalencia puede permitir la producción de nuevas frases, y sentar las bases para una interpretación comportamental de la generatividad del lenguaje" (Liddy, BarnesHolmes \& Hampson, 2001, p. 411).

Aunque se considera que la transferencia de función y la formación de clases de equivalencia son insuficientes para explicar por sí mismas la complejidad de la gramática del lenguaje, se propone que estos fenómenos pueden resultar relevantes en su adquisición y evolución, constituyendo prerrequisitos para su desarrollo. 
FIGURA 1

ESTRUCTURA DE LOS ENSAYOS DE ENTRENAMIENTO Y TEST DE LAS TAREAS DE RELACIONES DE EQUIVALENCIA Y GRAMÁTICA ARTIFICIAL

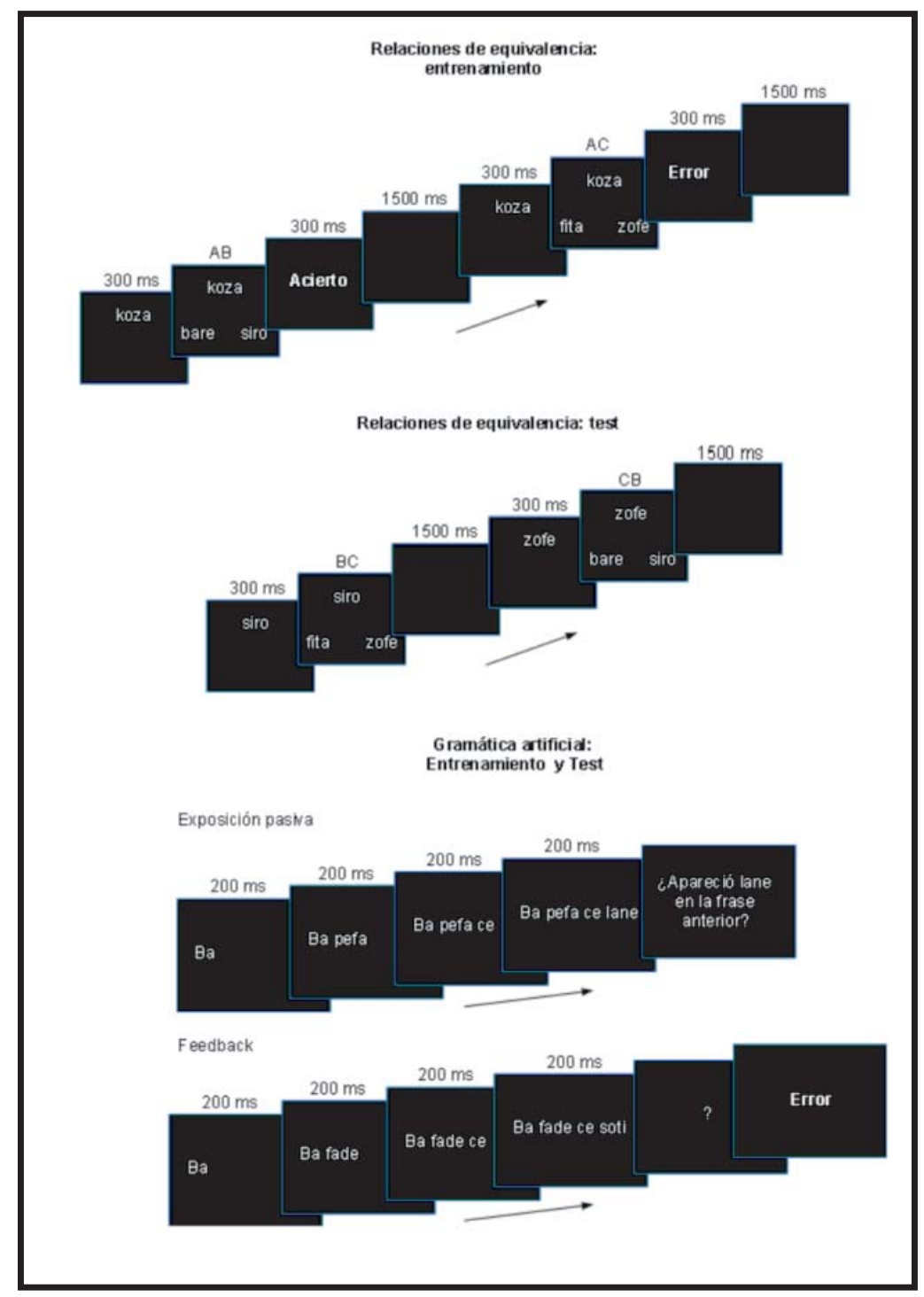

Nota:

Curso temporal de ensayos de entrenamiento de relaciones AB y AC (arriba).

Curso temporal de ensayos de test de las relaciones BC y CB (simetría - transitividad combinadas) (medio). Curso temporal de un ensayo de los bloques de exposición pasiva y un ensayo de los bloques con feedback en la tarea de gramática artificial (abajo).

Las secuencias son presentadas de izquierda a derecha, de a un ítem por vez. 
FIGURA 2

ESTRUCTURA DE LOS ENSAYOS DEL TEST DE TRANSFERENCIA DE FUNCIONES SINTÁCTICAS

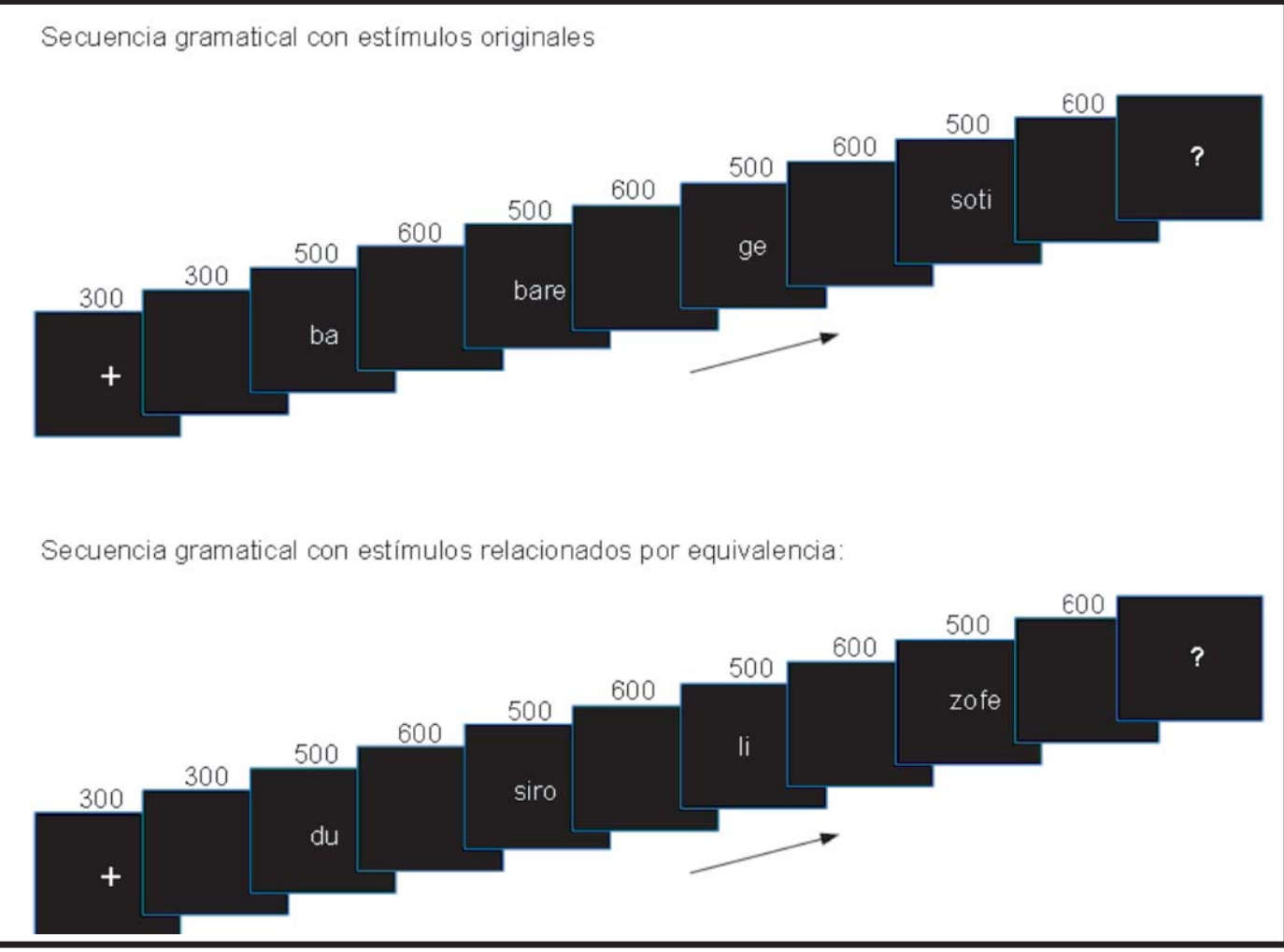

Nota:

Curso temporal de un ensayo en el que se presenta una secuencia gramatical construida con estímulos originales (arriba).

Curso temporal de un ensayo en el que se presenta una secuencia gramatical cuyo item final es un estímulo relacionado por equivalencia (abajo).

El sujeto responde si considera que la secuencia cumple con las reglas de la gramática al aparecer el signo "?". 


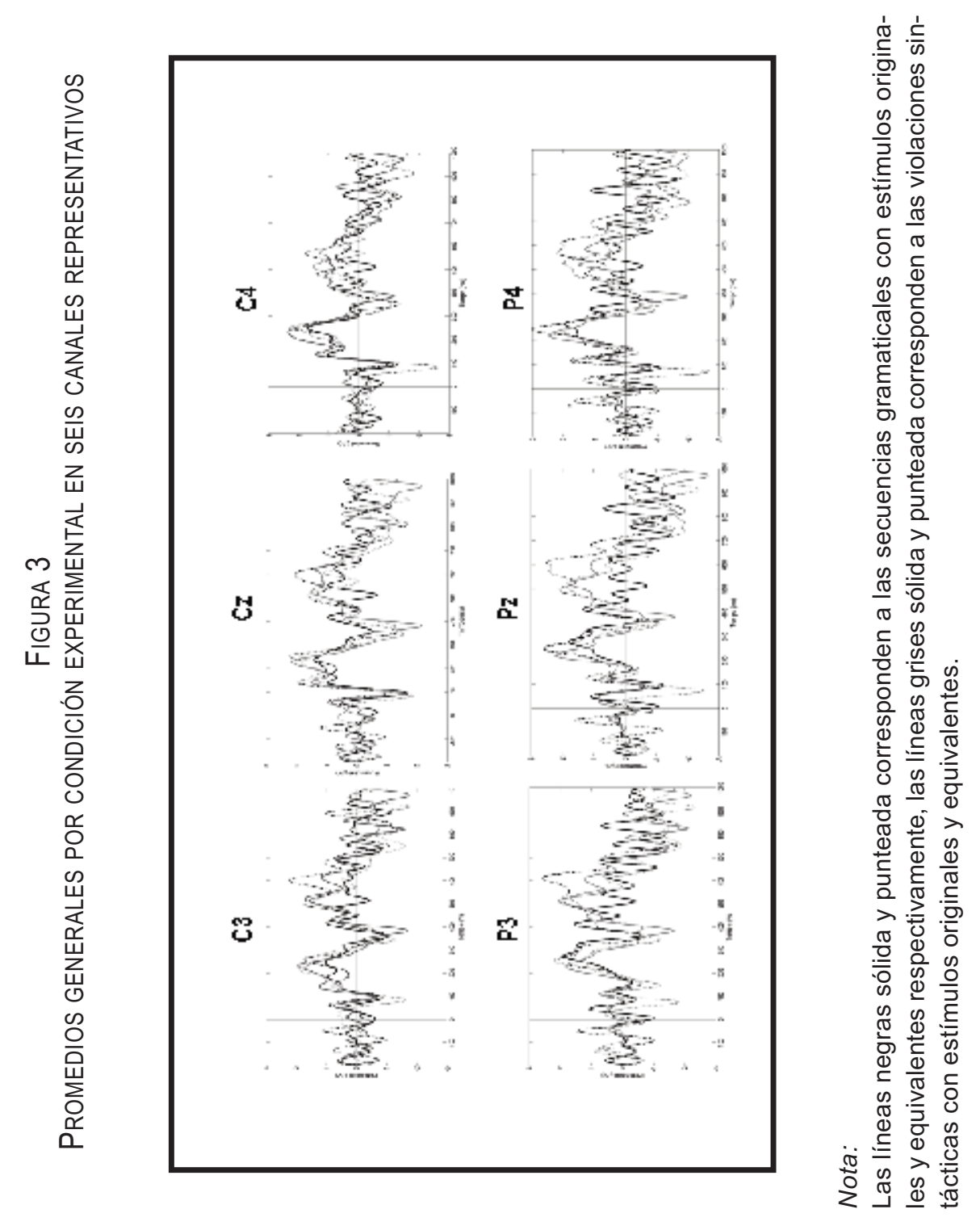




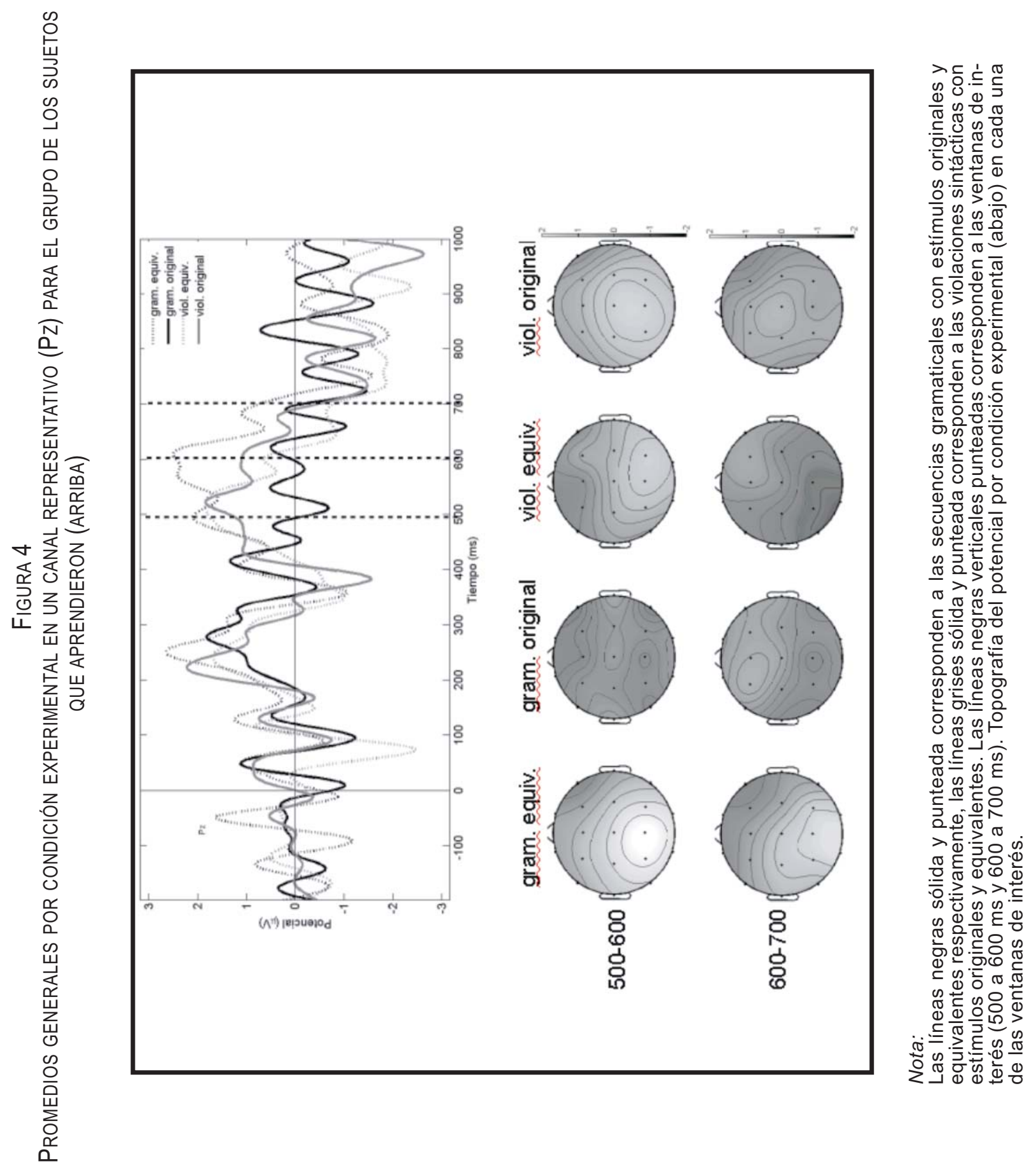


PREs en transferencia de funciones sintácticas

TABLA 1

EJEMPLOS DE ORACIONES GRAMATICALES Y VIOLACIONES SINTÁCTICAS CON ESTÍMULOS ORIGINALES Y RELACIONADOS POR EQUIVALENCIA

\begin{tabular}{|l|c|c|}
\hline \multicolumn{1}{|c|}{ Estímulos } & Gramatical & Violación \\
\hline Originales & $\begin{array}{c}\text { ba bare ge soti } \\
\text { du siro li lane }\end{array}$ & $\begin{array}{c}\text { ba leda ce bare } \\
\text { du voni ki } \underline{\text { soti }}\end{array}$ \\
$\begin{array}{l}\text { Relacionados por } \\
\text { equivalencia }\end{array}$ & $\begin{array}{l}\text { ba fita ge soti } \\
\text { du siro li zofe }\end{array}$ & du voni ki $\underline{\text { zofa }}$ \\
\end{tabular}

Notación:

Los estímulos relacionados por equivalencia que reemplazan a los originales aparecen en negrita.

Las violaciones aparecen subrayadas.

TABLA 2

PORCENTAJES DE ACIERTOS EN LAS FASES DE ENTRENAMIENTO EN EQUIVALENCIA Y GRAMÁTICA ARTIFICIAL Y EN EL TEST DE TRANSFERENCIA DE FUNCIONES SINTÁCTICAS (MEDIA Y DESVIIO ESTÁNDAR).

\begin{tabular}{|l|c|c|c|}
\hline \multicolumn{1}{|c|}{ Grupo } & $\begin{array}{c}\text { Entr. Equivalencia* } \\
\%\end{array}$ & $\begin{array}{c}\text { Entr. Gramática artificial** } \\
\%\end{array}$ & $\begin{array}{c}\text { Test } \\
\%\end{array}$ \\
\hline Superaron & $98.27 \pm 2.27$ & $72.74 \pm 17.64$ & $69.83 \pm 17.14$ \\
No superaron & $87.99 \pm 20.57$ & $59.44 \pm 12.14$ & $49.62 \pm 2.7$ \\
\hline
\end{tabular}

Nota:

*El porcentaje de aciertos en la tarea de equivalencia corresponde a la fase de test de relaciones BC-CB, en la que se verificó la formación de clases de equivalencia.

${ }^{* *}$ El porcentaje de aciertos en la tarea de gramática artificial corresponde al segundo bloque de entrenamiento con feedback. 
TABLA 3

PORCENTAJE DE ACIERTOS EN LA TAREA DE TEST, POR GRUPO Y CONDICIÓN EXPERIMENTAL (MEDIA Y DESVÍO ESTÁNDAR)

\begin{tabular}{|l|c|c|c|c|}
\hline \multicolumn{1}{|c|}{ Grupo } & $\begin{array}{c}\text { Gramática original } \\
\%\end{array}$ & $\begin{array}{c}\text { Gramática equiv. } \\
\%\end{array}$ & $\begin{array}{c}\text { Violación original } \\
\%\end{array}$ & Violación equiv. \\
\hline Superaron & $82.78 \pm 12.77$ & $66.94 \pm 19.71$ & $67.93 \pm 17.14$ & $61.67 \pm 22$ \\
No superaron & $59.58 \pm 8.57$ & $41.25 \pm 5.64$ & $52.5 \pm 10.25$ & $44.17 \pm 9.83$ \\
\hline
\end{tabular}

\section{REFERENCIAS BIBLIOGRÁFICAS}

Arismendi, M., Tabullo, A., Vernis, S., Primero, G., Fiorentini, L., Sánchez, F. et al. (2012). El fenómeno de transferencia en gramáticas artificiales: Efectos de la forma de entrenamiento [The phenomenon of transfer in artificial grammars: Effects of the form of workout]. Anuario de Investigaciones de la Facultad de Psicología $U B A, X I X, 319-125$.

Arntzen, E., Grondahl, T. \& Eilifsen, C. (2010). The effects of different training structures in the establishment of conditional discriminations and the subsequent performance on the tests for stimulus equivalence. The Psychological Record, 60, 437-461.

Arntzen, E. \& Holth, P. (2000). Differential probabilities of equivalence outcome in individual subjects as a function of training structure. The Psychological Record, 50, 603-628.

Aslin, R.N., Saffran, J.R. \& Newport, E.L. (1998). Computation of conditional probability statistics by 8-month old infants. Psychological Science, 9, 321-324.
Augustson, K.G. \& Dougher, M.J. (1992). Teaching conditional discrimination to young children: Some methodological successes and failures. Experimental Analysis of Human Behaviour Bulletin, 9, 21-24.

Bahlmann, J., Gunter, T.C. \& Friederici, A.D. (2006). Hierarchical and linear sequence processing: An electrophysiological exploration of two different grammar types. Journal of Cognitive Neuroscience, 18, 1829-1842. http://dx.doi. org/10.1162/jocn.2006.18.11.1829

Bahlmann, J., Schubotz, R.I. \& Friederici, A.D. (2008). Hierarchical artificial grammar processing engages Broca's area. NeuroImage, 42, 525-534. http://dx.doi.org/10.1016/j.neuroi ma ge.2008.04.249

Barnes, D., McCullagh, P.D. \& Keenan, M. (1990). Equivalence class formation in non-hearingimpaired children and hearing-impaired children. The Analysis of Verbal Behaviour, 8, 1930.

Barnes, D., Smeets, P.M. \& Leader, G. (1996). New procedures for establishing emergent matching performances in children and adults: Implication for stimulus equivalence. En T.R. Zentall 
\& P.M. Smeets (Eds.), Stimulus class formation in humans and animals. Amsterdam: Elsevier.

Barnes-Holmes, D., Regan, D., Barnes-Holmes, Y., Commins, S., Walsh, D., Stewart, I. et al. (2005). Derived stimulus relations, semantic priming, and event-related potentials: testing a behavioral theory of semantic networks. Journal of the Experimental Analysis of Behavior, 84, 37-84. http://dx.doi.org/10.1901/ jeab. 2005. 78-04

Brookhuis, K.A., Mulder, G., Mulder, L.J., Gloerich, A.B., van Dellen, H.J., van der Meere, J.J. et al. (1981). Late positive components and stimulus evaluation time. Biological Psychology, 13,107-123.

Chase, P.N., Ellenwood, D.W. \& Madden, G.J. (2008). A behavior analytic analogue of learning to use synonyms, syntax, and parts of speech. Analysis of Verbal Behavior, 24, 31-54.

Christiansen, M., Conway, C. \& Onnis, L. (2012). Similar neural correlates for language and sequential learning: Evidence from event-related brain potentials. Language and Cognitive Processes, 27, 231-256. http://dx.doi.org/10. 1080/01690965.2011.606666

Coulson, S., King, J.W. \& Kutas, M. (1998). Expect the unexpected: Event-related brain response to morphosyntactic violations. Language and Cognitive Processes, 13, 21-58.

Cullinan, V., Barnes, D., Hampson, P.J. \& Lyddy, F. (1994). A transfer of explicitly and non-explicitly trained sequence responses through equivalence relations: An experimental demonstration and connectionist model. The Psychological Record, 44, 559-586.

Delorme, A. \& Makeig, S. (2004). EEGLAB: An open source toolbox for analysis of single-trial EEG dynamics. The Journal of Neuroscience Methods, 134, 9-21.

DeLong, K.A., Urbach, T.P. \& Kutas, M. (2005). Probabilistic word pre-activation during language comprehension inferred from electrical brain activity. Nature Neuroscience, 8(8), 11171121. http://dx.doi.org/10.1038/nn1504
Devany, J.M., Hayes, S.C. \& Nelson, R.O. (1986). Equivalence class formation in language-able and language-disabled children. Journal of the Experimental Analysis of Behavior, 46, 243257.

Dickins, T.E. \& Dickins, D.W. (2001). Symbols, stimulus equivalence and the origins of language. Behavior and Philosophy; 29, 221-244.

Donchin, E. \& Coles, M.G. (1988). Is the P300 component a manifestation of context updating? Behavioral and Brain Sciences, 11(3), 357-427.

Dugdale, N. \& Lowe, C.F. (2000). Testing for symmetry in the conditional discriminations of language trained chimpanzees. Journal of the Experimental Analysis of Behavior, 73, 5-22.

Fiser, J. \& Aslin, R.N. (2002). Statistical learning of higher-order temporal structure from visual shape-sequences. Journal of Experimental Psychology: Learning, Memory, and Cognition, 28(3), 458-467.

Folia, V., Forkstam, C., Ingvar, M., Hagoort, P. \& Petersson, K.M. (2011). Implicit artificial syntax processing: Genes, preference, and bounded recursion. Biolinguistics, 5(1/2), 105-132.

Gibson, E. (1998). Linguistic complexity: Locality of syntactic dependencies. Cognition, 68, 1-76.

Gómez, R.L. \& Gerken, L.A. (1999). Artificial grammar learning by one-year-olds leads to specific and abstract knowledge. Cognition, 70, 109-135.

Hagoort, P. (2009). Reflections on the neurobiology of syntax. En D. Bickerton \& E. Szathmáry (Eds.), Biological foundations and origin of syntax. Strungmann Forum reports (Vol. 3, pp. 279-296). Cambridge, MA: MIT Press.

Hahne, A. \& Friederici, A.D. (1999). Electrophysiological evidence for two steps in syntactic analysis. Early automatic and late controlled processes. Journal of Cognitive Neuroscience, 11(2), 194-205.

Hall, G. (1996). Learning about associatively activated representations: Implications for acquired 
equivalence and perceptual learning. Animal Learning \& Behavior, 24, 233-255.

Hayes, S.C. (1992). Verbal relations, cognition, and the evolution of behaviour analysis. American Psychologist, 47, 1383-1395.

Hayes, S.C., Barnes-Holmes, D. \& Roche, B. (Eds.). (2001). Relational frame theory: A PostSkinnerian account of human language and cognition. New York: Plenum Press.

Hinojosa, J.A., Martin-Loeches, M., Casado, P., Muñoz, F. \& Rubia, F.J. (2003). Similarities and differences between phrase structure and morphosyntactic violations in Spanish: An eventrelated potentials study. Language and Cognitive Processes, 18(2), 113-142.

Horne, P.J. \& Lowe, C.F. (1996). On the origins of naming and other symbolic behavior. Journal of the Experimental Analysis of Behavior, 65, 185-241.

Imam, A.A. \& Chase, P.N. (1988). A stimulus equivalence model of syntactic classes. The Mexican Journal of Behavior Analysis, 14, 1121.

Kaan, E. (2007). Event-related potentials and language processing. A brief introduction. Language and Linguistics Compass, 1(6), 572-591.

Kaan, E. (2009). Fundamental syntactic phenomena and their putative relation to the brain. En D. Bickerton \& E. Szathmáry (Eds.), Biological foundations and origin of syntax: Strüngmann forum reports (Vol. 3, pp. 117-133). Cambridge, MA: MIT Press.

Kaan, E., Harris, A., Gibson, E. \& Holcomb, P. (2000). The P600 as an index of syntactic integration difficulty. Language and Cognitive Processes, 15, 159-201.

Keller, F.S. \& Schoenfeld, W.N. (1950). Principles of psychology. New York: Appleton-CenturyCrofts.

Kutas, M. \& Hillyard, S.A. (1980). Event-related potentials to semantically inappropriate and surprisingly large words. Biological Psychology, 11, 99-116.
Kutas, M., McCarthy, G. \& Donchin, E. (1977). Augmenting mental chronometry: The P300 as a measure of stimulus evaluation time. Science, 197, 792-795.

Lazar, R.M. (1977). Extending sequence-class membership with matching to sample. Journal of the Experimental Analysis of Behavior, 27, 381-392.

Lazar, R.M. \& Kotlarchyk, B.J. (1986). Secondorder control of sequence-class equivalences in children. Behavioral Processes, 13, 205-215.

Liddy, F., Barnes-Holmes, D. \& Hampson, P.J. (2001). A transfer of sequence function via equivalence in a connectionist network. The Psychological Record, 51, 409-428.

Maye, J., Werker, J. \& Gerken, L.A. (2002). Infant sensitivity to distributional information can affect phonetic discrimination. Cognition, 82, 101-111.

Mognon, A., Jovicich, J., Bruzzone, L. \& Buiatti, M. (2010). ADJUST: An automatic EEG artifact detector based on the joint use of spatial and temporal features. Psychophysiology, 48, 229-240.

Moreno, E.M., Federmeier, K.D. \& Kutas, M. (2002). Switching languages, switching palabras (words): An electrophysiological study of code switching. Brain \& Language, 80, 188207.

Moreno-Montes, E.M. (2005). Estudios electrofisiológicos del procesamiento de cambios de idioma en bilingües [Electrophysiological studies of language switching processing in bilingual subjetcs]. Tesis doctoral no publicada. Universidad Complutense de Madrid. España.

Münte, T.F., Matzke, M. \& Johannes, S. (1997). Brain activity associated with syntactic incongruencies in words and pseudo-words. Journal of Cognitive Neuroscience, 93, 318-329.

Osterhout, L., Bersick, M. \& McLaughlin, J. (1997). Brain potentials reflect violations of gender stereotypes. Memory and Cognition, 25(3), 273-285. 
Osterhout, L. \& Holcomb, P.J. (1992). Event-related brain potentials elicited by syntactic anomaly. Journal of Memory and Language, 31, 785806.

Patel, A.D., Gibson, E., Ratner, J., Besson, M. \& Holcomb, P.J. (1998). Processing syntactic relations in language and music: An event-related potential study. Journal of Cognitive Neuroscience, 10, 717-733.

Peña, M., Bonnatti, L., Nespor, M. \& Mehler, J. (2002). Signal-driven computations in speech processing. Science, 298, 604-607.

Petersson, K.M., Folia, V. \& Hagoort, P. (2012). What artificial grammar learning reveals about the neurobiology of syntax? Brain \& Language, 120, 83-95. http://dx.doi.org/10.1016/j.bandl. 2010.08.003

Petersson, K.M., Forkstam, C. \& Ingvar, M. (2004). Artificial syntactic violations activate Broca's region. Cognitive Science, 28(3), 383407. http://dx.doi.org/10.1207/s15516 709cog 2803

Reber, A.S. (1967). Implicit learning of artificial grammars. Journal of Verbal Learning and Behavior, 6, 855-863.

Reber, A.S. (1989). Implicit learning and tacit knowledge. Journal of Experimental Psychology: General, 118, 219-235.

Reber, A.S. (1993). Implicit learning and tacit knowledge: An essay on the cognitive unconscious. London: Oxford University Press.

Roche, B. \& Barnes, D. (1997). A transformation of respondently conditioned stimulus function in accordance with arbitrarily applicable relations. Journal of the Experimental Analysis of Behavior, 67, 275-300.

Saffran, J.R. (2001).Words in a sea of sounds: The output of infant statistical learning. Cognition, 81, 149-169.

Saffran, J.R. (2002). Constraints on statistical language learning. Journal of Memory and Language, 47, 172-196.
Saffran, J.R., Aslin, R.N. \& Newport, E.L. (1996). Statistical learning by 8-month-old infants. Science, 274, 1926-1928.

Sidman, M. (1986). Functional analysis of emergent verbal classes. En T. Thompson, N. Dugdale \& M.D. Zeiler (Eds.), Analysis and integration of behavioral units. Hillsdale, NJ: Erlbaum.

Sidman, M. (1994). Equivalence relations and behavior: A research story. Boston: Authors Cooperative.

Sidman, M. \& Tailby, W. (1982). Conditional discrimination vs. matching to sample: An expansion of the testing paradigm. Journal of the Experimental Analysis of Behavior, 37, 522.

Sommer, W., Leuthold, H. \& Matt, J. (1998). The expectancies that govern the P300 amplitude are mostly automatic and unconscious. Behavioral and Brain Sciences. Continuing Commentary, 21, 149-168.

Tabullo, A., Sevilla, Y., Pasqualetti, G., Vernis, S., Segura, E. et al. (2011). Expectancy modulates a late positive ERP in an artificial grammar task. Brain Research, 1373, 131-143. http://dx.doi. org/10.1016/j.brainres.2010.12.0 23

Tabullo, A., Sevilla, Y., Segura, E., Zanutto, S. \& Wainselboim, A. (2013). An ERP study of structural anomalies in native and semantic free artificial grammar: Evidence for shared processing mechanisms. Brain Research, 1527, 149-160. http://dx.doi.org/10.1016/j.brainres. 2013.05.022

Thomas, M.S.C. \& Allport, A. (2000). Language switching costs in bilingual visual word recognition. Journal of Memory \& Language, 43(1), 44-66.

Thompson, S. \& Newport, E. (2007). Statistical learning of syntax: The role of transitional probability. Language Learning and Development, 3, 1-42. http://dx.doi.org/10.1080/154 75440709336999 
Van Berkum, J.J.A., Brown, C.M., Zwitserlood, P., Kooijman, V. \& Hagoort, P. (2005). Anticipating upcoming words in discourse: Evidence from ERPs and reading times. Journal of Experimental Psychology: Learning, Memory, and Cognition, 31(3), 443-467.
Wulfert, E. \& Hayes, S.C. (1988). Transfer of conditional ordering response through conditional equivalence classes. Journal of Experimental Analysis of Behavior, 50, 125-144.

Laboratorio de Biología del Comportamiento Instituto de Biología y Medicina Experimental (IBYME) Consejo Nacional de Investigaciones Científicas y Técnicas (CONICET) Ciudad Autónoma de Buenos Aires República Argentina

Fecha de recepción: 16 de junio de 2014 Fecha de aceptación: 11 de noviembre de 2014 